\title{
Improving medication safety in varied health systems
}

\author{
Chui, Michelle A.
}

2019-07

Chui , M A , Pohjanoksa-Mäntylä, M \& Snyder , M E 2019 , ' Improving medication safety in varied health systems ' , Research in Social and Administrative Pharmacy, vol. 15 , no. 7 , pp. 811-812 . https://doi.org/10.1016/j.sapharm.2019.04.012

http://hdl.handle.net/10138/317188

https://doi.org/10.1016/j.sapharm.2019.04.012

cc_by_nc_nd

acceptedVersion

Downloaded from Helda, University of Helsinki institutional repository.

This is an electronic reprint of the original article.

This reprint may differ from the original in pagination and typographic detail.

Please cite the original version. 
Improving medication safety in varied health systems

Dr. Michelle A. Chui, PharmD, PhD, FAPhA. Associate Professor \& Vice-Chair, Social \& Administrative Sciences Division; Director, Sonderegger Research Center for Improved Medication Outcomes; University of Wisconsin - Madison. michelle.chui@wisc.edu

Dr. Marika Pohjanoksa-Mäntylä, PhD, MSc (Pharm). Senior Lecturer, Clinical Pharmacy Group, Division of Pharmacology and Pharmacotherapy, Faculty of Pharmacy, University of Helsinki, Finland. marika.pohjanoksa@helsinki.fi

Dr. Margie E. Snyder, PharmD, MPH, FCCP. Associate Professor of Pharmacy Practice, Purdue University College of Pharmacy. snyderme@purdue.edu

Since the Institute of Medicine (now National Academies of Science, Engineering, and Medicine) report of $1999,{ }^{1}$ patient safety work in inpatient settings, surgery, emergency department, devices, and infection control has been advanced. Many studies have been done to identify hazards and contributing factors (e.g., organizational culture, inter-professional communication). And, many improvements have been implemented to reduce those hazards. ${ }^{2} \mathrm{~A}$ unique and distinct component of patient safety as a whole is medication safety. But, unlike many advances in inpatient medical environments, medication safety (particularly in the outpatient setting) has lagged behind, in part because the medication use process is diametrically different in inpatient and outpatient settings. In the outpatient setting, patients and caregivers must assume significant responsibility in their own medication administration; therefore, the hazards that lead to medication errors are multi-dimensional and difficult to quantify.

Medication errors are among the most common incidents leading to adverse events in patient care and result in considerable human suffering and unnecessary deaths. As an estimate, one medication error occurs per hospitalized patient daily, and medication errors cost tens of billions of US dollars each year. Medication errors can occur in any healthcare setting during various stages of the medication use process, including prescribing, dispensing, administering and using medicines. It has been well established that the majority of the errors would be preventable and commonly provoked by weaknesses in organizational systems and processes (i.e., system factors).

Fifteen years after the first IOM report To Err is Human, the National Patient Safety Foundation made eight recommendations for achieving total system safety, including creating a common set of safety metrics that reflect meaningful outcomes, establishing and sustaining a culture of safety, centralizing oversight of patient safety at the national level, improving the safety of information technology, and supporting patients, families, and the health care workforce. ${ }^{3}$

In 2017, The World Health Organization (WHO) launched the Global Patient Safety Challenge on Medication Safety with a vision/goal to reduce the level of severe, avoidable harm related to medications by $50 \%$ over the next five years throughout the world. ${ }^{4}$ The specific objectives of the challenge include: 1 ) To assess the scope and nature of avoidable harm and strengthen the monitoring systems to detect and track this harm; 2) To create a framework for action aimed at patients, health professionals and policy makers, to facilitate improvements in ordering, prescribing, preparation, dispensing, administration and 
monitoring practices, which can be adopted and adapted locally; 3) To develop guidance, materials, technologies and tools to support the setting up of safer medication use systems for reducing medication errors; 4) To engage key stakeholders, partners and industry to raise awareness of the problem and actively pursue efforts to improve medication safety; and 5) To empower families and their caregivers to become actively involved and engaged in treatment or care decisions, ask questions, spot errors and effectively manage their medications.

Medication errors and unsafe medication practices are a global public health issue. Despite myriad initiatives undertaken internationally, nationally and locally to improve patient safety, there is still much to do in this area. As a discipline, medication-focused safety science is in its adolescence, and is still maturing and coalescing into a cohesive body of evidence-based, generalizable, and scalable research. As a result, Research in Social and Administrative Pharmacy issued a call for papers toward a special issue on medication safety. It was believed that a special issue could engage pharmacists, researchers, and policy makers in this important dialogue of how to move forward together in a thoughtful way. It is hoped that the readers of this special issue come away with an appreciation of the work that has already been done, but to heighten their awareness that much work is still to come, and that the need to understand how medication errors take place, and to develop and evaluate creative and evidence-based interventions that will improve the safety of the patients that we care for.

Within this special issue will be found fourteen manuscripts - one editorial, five commentaries and nine original research articles. This issue leads with an editorial about Dr. Maria Cordina from the World Health Organization, highlighting the urgency to prepare the health workforce to challenging task of improving medication safety. ${ }^{5}$ It then provides an overview and cross-section of patient safety research before and immediately after the launch of WHO Global Patient Safety Challenge. You will find original research from a diverse group of countries, representing different health systems and policies, from North America to Europe to China. Medication safety research is approached from both quantitative methods with large population level data, as well as a deep dive into context specific details using qualitative and mixed methods.

All commentaries raise the need for joint actions to achieve the ambitious aim of WHO Global Patient Safety Challenge to reduce medication errors and medication related harm by $50 \%$ in the following 5 years throughout the world. The Bader et al., Smith et al. and Hong et al. provides community pharmacy perspective to this themed issue. ${ }^{6-8}$ They highlight that pharmacists have a key role in preventing and decreasing risks in medicines use, and thus, mitigating the global challenge of patient safety. Additionally, the Bader et al. describes how the medication safety is a strategic priority of International Pharmaceutical Federation (FIP), and how FIP have and will contribute to this challenge in close collaboration with the World Health Organization (WHO). Whereas, Smith presents a novel framework for how to move forward and to promote primary care medication use and safety as part of preventative care or chronic care management initiatives. Tetteh provides perspectives from high and low-income countries, and upraises the need to take account national needs and challenges when outlining the implementation strategies of Global Patient Safety Challenge in different countries. ${ }^{9}$ For example in US, as described by Abraham et al., misuse of prescription opioids and associated negative health outcomes is a leading public health crisis among adolescents. ${ }^{10}$

Four original research articles, with work contributed from China, Finland, and the United States, describe the important role of medication error reporting systems. Chen et al. describes the effect of 
implementation of a Medication Error Monitoring System at one hospital in China on the number and types of medication errors reported by physicians and pharmacists. ${ }^{11}$ Lester et al. and Darbishire et al. described medication error reporting in community pharmacy settings in the United States. ${ }^{12-13}$ Lester et al. applied text analysis to the narratives submitted with medication error reports in order to expand on information provided in the structured sections of reports. Darbishire et al. engaged second professional year pharmacy students to collect data on medication error reporting as part of their experiential learning experiences at community pharmacies. These papers provide insights into novel strategies for collecting data about medication errors and reporting practices. However, Holmstrom et al. demonstrate that more work is needed to ensure consistency in error classification among professionals submitting error reports. ${ }^{14}$

Finally, four original research articles describe tools and strategies for error prevention in Australia, Canada, Finland, and the United States. Schepel et al. documents how clinical pharmacy services in Finnish hospitals changed from 2011 to 2016 in response to national patient safety priorities. ${ }^{15}$ Also focused on institutional practice, Marwitz et al. explores IV smart pump alert overrides for both high alert and nonhigh alert medications, determining that work is needed to reduce alert fatigue for smart pump users. ${ }^{16}$ Barker et al. and Wood et al. present data pertaining to community pharmacy practice. ${ }^{17-18}$ Barker et al. interviewed Canadian community pharmacists to identify barriers to use of patient safety information sources. Wood et al. describes the implementation of clinical decision support tool for pharmacists and also found some barriers to use.

Patient safety science is making significant advances toward reducing medication errors and improving health care quality. Indeed, the manuscripts presented in this issue exemplify the efforts that are being made globally. But progress continues to be slow, in part because the patient safety challenge is multidimensional and multi-faceted. It is our hope that this issue will add to the urgent need for greater investment in patient safety, and specifically encourage discussion and research to follow implementation and outcomes of medication safety initiatives.

\section{References}

1 Wakefield, M. (2000). To err is human: An Institute of Medicine report. Professional Psychology: Research and Practice, 31(3), 243-244. http://dx.doi.org/10.1037/h0092814

2 Shekelle PG et al. The top patient safety strategies that can be encouraged for adoption now. Annals of Internal Medicine. 2013; 158(5_Part_2): 365-8. DOI: 10.7326/0003-4819-158-5-201303051-00001

3 National Patient Safety Foundation. Free from harm: accelerating patient safety improvement fifteen years after To Err is Human. www.npsf/free-from-harm.

4 World Health Organization. Medication without harm. https://www.who.int/patientsafety/medicationsafety/en/

5 Cordina M. Preparing the health workforce to enhance medication safety. Research in Social and Administrative Pharmacy. Res Social Adm Pharm. 2019 Mar 13. pii: S1551-7411(19)30095-6. doi: 10.1016/j.sapharm.2019.03.002. 
6 Bader L, Kusynová Z, Duggan C. FIP Perspectives: Realising global patient safety goals requires an integrated approach with pharmacy at the core. Res Social Adm Pharm. 2019 Feb 23. pii: S15517411(19)30129-9. doi: 10.1016/j.sapharm.2019.02.012.

7 Smith M: No more status quo: A multi-dimensional framework for primary care medication use and safety. Res Social Adm Pharm. 2018 Oct 1. pii: S1551-7411(18)30709-5. doi: 10.1016/j.sapharm.2018.09.011.

8 Hong K, Hong YD, Cooke CE. Medication Errors in Community Pharmacies: The Need for Commitment, Transparency, and Research. Res Social Adm Pharm. 2018 Dec 1. pii: S1551-7411(18)30719-8. doi: 10.1016/j.sapharm.2018.11.014.

9 Tetteh EK. Reducing Avoidable Medication Related Harm: What will it take? Res Social Adm Pharm. 2019 Apr 5. pii: S1551-7411(18)30658-2. doi: 10.1016/j.sapharm.2019.04.002.

10 Abraham O, Thakur T, Brown R. Prescription opioid misuse and the need to promote medication safety among adolescents. Res Social Adm Pharm. 2019 Jan 11. pii: S1551-7411(18)30595-3. doi: 10.1016/j.sapharm.2019.01.003.

11 Chen Y, Wu X, Huang Z, Lin W, Li Y, Yang J, Li J. Evaluation of a medication error monitoring system to reduce the incidence of medication errors in a clinical setting. Res Social Adm Pharm. 2019 Feb 21. pii: S1551-7411(18)30390-5. doi: 10.1016/j.sapharm.2019.02.006.

12 Lester CA, Kessler JM, Modisett T, Chui MA. A text mining analysis of medication quality related event reports from community pharmacies. Res Social Adm Pharm. 2018 Sep 26. pii: S1551-7411(18)30281-X. doi: 10.1016/j.sapharm.2018.09.013.

13 Darbishire PL, Zhao JC, Sodhi A, Anderson CM. Student observation of medication error reporting practices in community pharmacy setting. Res Social Adm Pharm. 2019 Mar 3. pii: S15517411(18)30735-6. doi: 10.1016/j.sapharm.2019.02.009.

14 Holmström AR, Järvinen R, Laaksonen R, Keistinen T, Doupi P, Airaksinen M: Inter-rater Reliability of Medication Error Classification in a Voluntary Patient Safety Incident Reporting System HaiPro in Finland. Res Social Adm Pharm. 2018 Nov 28. pii: S1551-7411(18)30694-6. doi: 10.1016/j.sapharm.2018.11.013.

15 Schepel L, Aronpuro K, Kvarnstrom K, et al. Strategies for improving medication safety in hospitals: evolution of clinical pharmacy services. Res Social Adm Pharm. 2019 Mar 16. pii: S1551-7411(18)306740. doi: 10.1016/j.sapharm.2019.02.004.

16 Martwitz KK, Giuliano KK, Su WT, Degnan D, Zink RJ, DeLaurentis P. High-Alert Medication Administration and Intravenous Smart Pumps: A Descriptive Analysis of Clinical Practice. Res Social Adm Pharm. 2019 Feb 22. pii: S1551-7411(18)30720-4. doi: 10.1016/j.sapharm.2019.02.007.

17 Barker JR, Boyle TC, Tayl L, et al. Barriers to the use of patient safety information sources by community pharmacies. Res Social Adm Pharm. 2019 Feb 27. pii: S1551-7411(18)30746-0. doi: 10.1016/j.sapharm.2019.02.015. 
18 Wood P, Tucci J, Anderson K, Mnatzaganian G. Implementation of a clinical tool to assess and address pain management requests in the pharmacy. Res Social Adm Pharm. 2018 Dec 30. pii: S1551-

7411(18)30702-2. doi: 10.1016/j.sapharm.2018.12.009. 